\title{
LIPSCHITZ POLYTOPES OF POSETS AND PERMUTATION STATISTICS
}

\author{
RAMAN SANYAL AND CHRISTIAN STUMP
}

\begin{abstract}
We introduce Lipschitz functions on a finite partially ordered set $P$ and study the associated Lipschitz polytope $\mathscr{L}(P)$. The geometry of $\mathscr{L}(P)$ can be described in terms of descent-compatible permutations and permutation statistics that generalize descents and big ascents. For ranked posets, Lipschitz polytopes are centrally-symmetric and Gorenstein, which implies symmetry and unimodality of the statistics. Finally, we define $(P, k)$-hypersimplices as generalizations of classical hypersimplices and give combinatorial interpretations of their volumes and $h^{*}$-vectors.
\end{abstract}

\section{INTRODUCTION}

Let $(P, \preceq)$ a finite partially ordered set (or poset, for short). A function $f: P \rightarrow \mathbb{R}$ is isotone or order preserving if

$$
f(a) \leq f(b) \text { whenever } a \preceq b .
$$

The order cone $\mathcal{K}(P)$ of $P$ is the collection of nonnegative isotone functions. The order cone is a gateway for a geometric perspective on enumerative problems on posets. The interplay of combinatorics and geometry is, in particular, fueled by analogies to continues mathematics. For example, Stanley's order polytope [17] is the set

$$
\mathcal{O}(P)=\left\{f \in \mathcal{K}(P):\|f\|_{\infty} \leq 1\right\}
$$

where $\|f\|_{\infty}=\max \{f(a): a \in P\}$. The theory of $P$-partitions concerns those $f \in \mathcal{K}(P)$ with $\|f\|_{1}=\sum_{a} f(a)=m$ for some fixed $m$. In this paper, we want to further the analogies to continuous functions. For two elements $a, b \in P$, we denote the minimal length of a saturated (or unrefineable) chain from $a$ to $b$ by $d_{P}(a, b)$ and set $d_{P}(a, b):=\infty$ if $a \npreceq b$. Then $d_{P}$ is a quasi-metric on $P$. An isotone function $f:(P, \preceq) \rightarrow \mathbb{R}$ is $\boldsymbol{k}$-Lipschitz if

$$
f(b)-f(a) \leq k \cdot d_{P}(a, b)
$$

for all $a \preceq b$. We say that a function $f$ is Lipschitz if $f$ is 1-Lipschitz. Let us write $\breve{P}$ for the poset obtained from $P$ by adjoining a minimum $\widehat{0}$. The collection $\widetilde{\mathscr{L}}(\breve{P})$ of isotone Lipschitz functions on $\check{P}$ is naturally an unbounded polyhedron and $k$-Lipschitz functions are precisely the elements in $k \cdot \widetilde{\mathscr{L}}(\breve{P})$. The lineality space of $\widetilde{\mathscr{L}}(\breve{P})$ is given by all constant functions and we define the Lipschitz polytope of $P$ as

$$
\mathscr{L}(P):=\{f \in \mathcal{K}(\check{P}): f \text { Lipschitz, } f(\widehat{0})=0\} .
$$

Concretely, the Lipschitz polytope of $(P, \preceq)$ is given by

$$
\mathscr{L}(P)=\left\{f \in \mathbb{R}^{P}: \begin{array}{ll}
0 \leq f(a) \leq 1 & \text { for } a \in \min P \\
0 \leq f(b)-f(a) \leq 1 & \text { for } a \prec \cdot b
\end{array}\right\},
$$

where $a \prec \cdot b$ denotes the cover relations of $P$.

A different motivation for the study of $\mathscr{L}(P)$ comes from $G$-Shi arrangements. The Hasse diagram of $\check{P}$ is the directed graph $G$ on nodes $\check{P}$ with $\operatorname{arcs}(a, b)$ whenever $a \prec \cdot b$ is a cover

Date: August 23, 2018.

2010 Mathematics Subject Classification. 06A07, 05A05, 52B12.

Key words and phrases. posets, isotone functions, Lipschitz polytopes, lattice polytopes, Gorenstein polytopes, permutation statistics.

R. Sanyal was supported by the DFG-Collaborative Research Center, TRR 109 "Discretization in Geometry and Dynamics". C. Stump was supported by the DFG grant STU 563/2 "Coxeter-Catalan combinatorics". 
relation. The corresponding $\boldsymbol{G}$-Shi arrangement is the arrangement of affine hyperplanes $\left\{x_{b}-x_{a}=0\right\}$ and $\left\{x_{b}-x_{a}=1\right\}$ for $a \prec \cdot b$. The $G$-Shi arrangements generalize the classical Shi arrangements [15, Ch. 7] and naturally occur in the geometric combinatorics of parking functions and spanning trees; see [7]. The Lipschitz polytope $\mathscr{L}(P)$ is thus a particular (relatively) bounded region of the $G$-Shi arrangement associated to the Hasse diagram of $P$.

We give some basic geometric properties of Lipschitz polytopes in Section 2 and, in particular, show that $\mathscr{L}(P)$ is always a lattice polytope. Hence, the function

$$
E(\mathscr{L}(P), k):=\left|\mathscr{L}(P) \cap \mathbb{Z}^{P}\right|
$$

counting integer-valued $k$-Lipschitz functions agrees with a polynomial of degree $|P|$. In Section 3, we describe the canonical regular and unimodular triangulation of $\mathscr{L}(P)$. Similar to the study of order polytopes, the simplices of the triangulation of $\mathscr{L}(P)$ can be described in terms of certain permutations of $P$. A descent-compatible permutation is a labeling of $P$ such that the number of descents along any saturated increasing chain with fixed endpoints is constant. The $h^{*}$-vector of $\mathscr{L}(P)$ can be determined in terms of the combinatorics of $(P, \preceq)$ and defines an ascent-type statistic on the collection of all descent-compatible permutations of $P$ If the Hasse diagram of $P$ is a rooted tree, then all permutations are descent-compatible and the statistic corresponds to big ascents, i.e. ascents that with step size at least 2 .

For posets $P$ such that $\check{P}$ is ranked, the Lipschitz polytope $\mathscr{L}(P)$ is centrally-symmetric and Gorenstein. Using results from the theory of lattice polytopes, we deduce in Section 4 that the statistic on descent-compatible permutations is symmetric and unimodal, similar to the classical descent statistic on all permutations.

For posets $P$ with a unique maximal element $\widehat{1}$, we define in Section 5 the $(P, k)$-hypersimplices. These are certain slaps of $\mathscr{L}(P)$ that generalize the well-known $(n, k)$-hypersimplices of [4]. In particular, the volumes of our $(P, k)$-hypersimplices can be interpreted as the number of descent-compatible permutations of $P$ with $k P$-descents. For a chain, this recovers the classical interpretation of volumes of $(n, k)$-hypersimplices as Eulerian numbers. In [10], Li gave an interpretation of the coefficient of $h^{*}$-vector of half-open $(n, k)$-hypersimplices as the exceedence statistic on permutations with $k$ descents and our approach to the $h^{*}$-vectors of Lipschitz polytopes via half-open decompositions yields a simple geometric proof of this fact.

\section{BASIC GEOMETRIC PROPERTIES}

In this section, we collect basic geometric properties of Lipschitz polytopes. To begin with, we put some examples on record.

Example 2.1 (Antichains and chains). If $P$ is an antichain, then $\mathscr{L}(P)=[0,1]^{P}$. On the other hand, if $P=[n]:=\{1, \ldots, n\}$ is a chain, then $\mathscr{L}(P)$ is also linearly isomorphic to $[0,1]^{P}$ under the map that takes $g \in[0,1]^{P}$ to the function $f:[n] \rightarrow \mathbb{R}$ with $f(i)=g(1)+\cdots+g(i)$.

Such a linear and lattice-preserving transformation from $\mathscr{L}(P)$ to a cube as in the previous example exists in other cases as well. Let us call a poset $P$ a rooted tree if $P$ has a unique minimal element and the Hasse diagram is a tree. For a connected poset, i.e., a poset for which the Hasse diagram is connected, this is equivalent to the property that for any $b \in P$, there is a unique $\bar{b} \in \check{P}$ with $\bar{b} \prec \cdot b$. Define $T_{P}: \mathbb{R}^{P} \rightarrow \mathbb{R}^{P}$ with $(T f)(b):=f(b)-f(\bar{b})$. This is an invertible linear and $\mathbb{Z}^{P}$-preserving transformation.

Proposition 2.2. Let $P$ be a rooted tree. Then $T(\mathscr{L}(P))=[0,1]^{P}$.

Proof. It follows from (1.1) that $0 \leq T f(b) \leq 1$ for all $b \in P$ and hence $T(\mathscr{L}(P)) \subseteq[0,1]^{P}$. For any $b \in P$, there is a unique maximal chain $\widehat{0}=c_{1} \prec \cdot \cdots \prec \cdot c_{k}=b$ and for $g \in \mathbb{R}^{P}$, it is easy to see that $\left(T^{-1} g\right)(b)=g\left(c_{1}\right)+\cdots+g\left(c_{k}\right)$. Thus, $\left(T^{-1} g\right)(\bar{b})=g\left(c_{1}\right)+\cdots+g\left(c_{k-1}\right)$ and $T^{-1}(g) \in \mathscr{L}(P)$ whenever $0 \leq g(b) \leq 1$ for all $b \in P$. This proves the claim. 
Example 2.3. The figure to the right, produced with the TikZ output of SageMath [20], illustrates $\mathscr{L}(P)$ for the following poset on 3 elements:
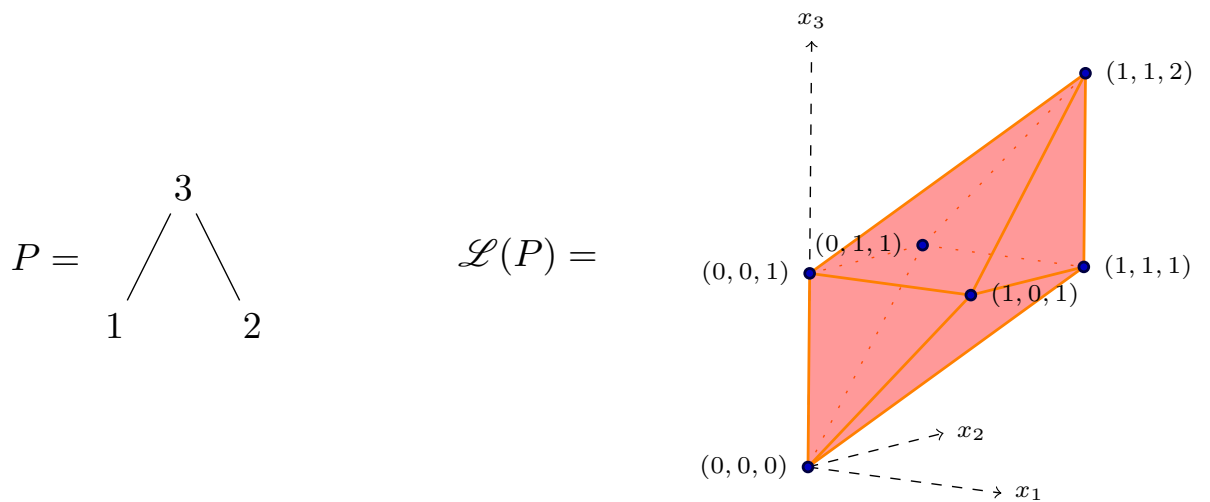

The following result is immediate and we will henceforth assume that all posets are connected.

Proposition 2.4. If $P=P_{1} \uplus P_{2}$, then $\mathscr{L}(P)=\mathscr{L}\left(P_{1}\right) \times \mathscr{L}\left(P_{2}\right)$.

Recall that $F \subseteq P$ is a filter if $a \in F$ and $a \preceq b$, implies $b \in F$. For a filter $F \subseteq P$, we denote by

$$
\mathcal{N}(F):=\{a \in \check{P} \backslash F: b \in F \text { for some } a \prec \cdot b\} \subseteq \check{P} .
$$

the neighborhood of $F$. In particular, $\widehat{0} \in \mathcal{N}(F)$ whenever $F$ contains a minimal element of $P$. A chain $\varnothing \neq F_{m} \subset \cdots \subset F_{1} \subseteq P$ of filters is neighbor-closed if $F_{i+1} \cup \mathcal{N}\left(F_{i+1}\right) \subseteq F_{i}$, or, equivalently, if

$$
a \notin F_{i} \quad \Longrightarrow \quad b \notin F_{i+1}
$$

for all $a, b \in \check{P}$ with $a \prec \cdot b$ and $1 \leq i<m$. With this notion, we have the following description of the vertices of Lipschitz polytopes.

Proposition 2.5. Let $P$ be a finite poset. Then $\mathbf{v} \in \mathbb{Z}^{P}$ is a vertex of $\mathscr{L}(P)$ if and only if

$$
\mathbf{v}=\mathbf{1}_{F_{m}}+\cdots+\mathbf{1}_{F_{1}}
$$

for a neighbor-closed chain $F_{m} \subset \cdots \subset F_{1} \subseteq P$ of nonempty filters in $P$.

Proof. Let us first observe that if $f \in \mathscr{L}(P) \cap \mathbb{Z}^{P}$, then $f(b)-f(a)=1$ or $f(b)-f(a)=0$ for all $\widehat{0} \preceq a \prec \cdot b$. In particular, this means that every lattice point can be uniquely recovered from the knowledge of which defining linear inequalities are satisfied with equality and hence the the vertices are the only lattice points of $\mathscr{L}(P)$. It therefore suffices to show that every lattice point of $\mathscr{L}(P)$ corresponds to a unique chain of filters as stated. This is quite standard: Let $\left\{t_{1}<\cdots<t_{m}\right\}$ be the distinct values of $f$ and define $F_{i}:=\left\{a \in P: f(a) \geq t_{i}\right\}$. Since $f$ is isotone, $F_{m} \subset \cdots \subset F_{1}$ is a chain of nonempty filters and

$$
f=\mathbf{1}_{F_{m}}+\cdots+\mathbf{1}_{F_{1}} .
$$

Now, $f \in \mathscr{L}(P)$ if and only if $f(b)-f(a) \leq 1$ for all $a \prec \cdot b$. That is, if there is at most one $F_{i}$ with $b \in F_{i}$ and $a \notin F_{i}$. This proves the claim.

Example 2.6. The Lipschitz polytopes of the short rooted tree on the left and the short hanging tree on the right
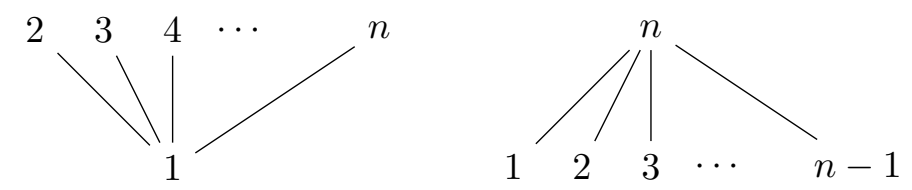
have $2 n$ and, respectively, $4(n-1)$ facets by construction. The short rooted tree has $2^{n}$ vertices in accordance with Proposition 2.2. These are given by $\mathbf{1}_{F}$ and $\mathbf{1}_{F}+\mathbf{1}_{[n]}$ for any subset $F \subseteq$ $\{2, \ldots, n\}$. On the other hand, the short hanging tree has $2^{n-1}+2$ vertices. These are given by $\mathbf{1}_{F}$ for any filter $F \subseteq[n]$, and $\mathbf{1}_{[n]}+\mathbf{1}_{\{n\}}$.

A full-dimensional polytope $\mathcal{P} \subset \mathbb{R}^{n}$ is 2-level if for every facet $F \subset \mathcal{P}$ there is a unique $\mathbf{q} \in \mathbb{R}^{n}$ such that the two parallel hyperplanes $\operatorname{aff}(F)$ and $\mathbf{q}+\operatorname{aff}(F)$ contain all vertices of $\mathcal{P}$. 2-level polytopes enjoy many favorable geometric properties; see, for example, [3, 5, 14]. As a corollary to the proof of Proposition 2.5, we note the following.

Corollary 2.7. Lipschitz polytopes are 2-level.

In particular, Sullivant [19] showed that every pulling triangulation of a 2-level polytope is unimodular. In the following section, we describe a unimodular triangulation of $\mathscr{L}(P)$ that is not of pulling-type. It might be interesting to study the combinatorial implications of pulling triangulations of Lipschitz polytopes.

\section{TRiangulations And VOlumes}

By definition, $\mathscr{L}(P)$ is an alcoved polytope in the sense of Lam-Postnikov [9]. This implies, that $\mathscr{L}(P)$ comes with a regular and unimodular triangulation and we will make use of this triangulation to compute the volumes and $h^{*}$-vectors of Lipschitz polytopes. We briefly recap the setup. One considers the affine braid arrangement in $\mathbb{R}^{n+1}$ given by the hyperplanes

$$
\left\{\mathbf{x} \in \mathbb{R}^{n+1}: x_{i}-x_{j}=\beta\right\} \quad \text { for } 0 \leq i<j \leq n \text { and } \beta \in \mathbb{Z} .
$$

The lineality space of the braid arrangement is $\mathbb{R} \cdot \mathbf{1}$ and the restriction to $\left\{x_{0}=0\right\} \cong \mathbb{R}^{n}$ yields an essential arrangement $\widetilde{\mathcal{A}}_{n}$ that decomposes $\mathbb{R}^{n}$ into infinitely bounded regions. Whenever convenient, we also refer to the 0 -th coordinate of a point $\mathbf{p} \in \mathbb{R}^{n}$ which, by construction, satisfies $p_{0}=0$.

Let $\mathbf{p} \in \mathbb{R}^{n}$ be a point not contained in any of the hyperplanes of $\widetilde{\mathcal{A}}_{n}$. Then $p_{i} \notin \mathbb{Z}$ for all $i$ and hence $\mathbf{p}=\mathbf{q}+\mathbf{r}$ with $\mathbf{q} \in \mathbb{Z}^{n}$ and $\mathbf{r} \in(0,1)^{n}$. Moreover, $p_{i}-p_{j} \notin \mathbb{Z}$ implies $r_{i} \neq r_{j}$ for $i \neq j$ and there is a unique permutation $\tau \in \mathfrak{S}_{n}$ such that

$$
0<r_{\hat{\tau}(1)}<r_{\hat{\tau}(2)}<\cdots<r_{\hat{\tau}(n)}<1
$$

where we write $\hat{\tau}:=\tau^{-1}$ for the inverse permutation. Define

$$
\Delta_{\tau}:=\left\{\mathbf{x} \in \mathbb{R}^{n}: 0 \leq x_{\hat{\tau}(1)} \leq x_{\hat{\tau}(2)} \leq \cdots \leq x_{\hat{\tau}(n)} \leq 1\right\} .
$$

This is an $n$-dimensional simplex with integral vertices and volume $\frac{1}{n !}$, i.e. $\Delta_{\tau}$ is a unimodular simplex with respect to the lattice $\mathbb{Z}^{n}$. The closed region containing $\mathbf{p}$ is given by $\mathbf{q}+\Delta_{\tau}$ and since $\mathbf{p}$ was arbitrary, all closed regions of $\tilde{\mathcal{A}}_{n}$ are of that form. In particular, any two regions are isomorphic with respect to a $\mathbb{Z}^{n}$-preserving affine transformation. The closed regions of $\tilde{\mathcal{A}}_{n}$ are called alcoves and a convex polytope $\mathcal{P} \subset \mathbb{R}^{n}$ is alcoved if it is the union of alcoves. Thus, an alcoved polytope naturally comes with a triangulation into unimodular simplices. Since the triangulation is induced by a hyperplane arrangement, it is regular.

We call a finite poset $(P, \preceq)$ naturally labeled if we identify its ground set with $\{1, \ldots, n\}$ for $n=|P|$ such that $a \prec b$ implies $a<b$. In this case, we can set $\breve{P}:=P \cup\{\widehat{0}:=0\}$ and with this labelling, it is clear that the Lipschitz polytope as defined in (1.1) is an alcoved polytope. We now determine the alcoves that compose $\mathscr{L}(P)$.

To later simplify notations, we set $\tau(0):=0$ for any permutation $\tau \in \mathfrak{S}_{n}$ and we recall that $q_{0}=0$ for any $\mathbf{q} \in \mathbb{R}^{n}$. We moreover define the descent set

$$
\operatorname{Des}(\tau):=\{0 \leq i<n: \tau(i)>\tau(i+1)\}
$$

and the inverse descent set by $\operatorname{iDes}(\tau):=\operatorname{Des}(\hat{\tau})$. Thus, $i \in \mathrm{iDes}(\tau)$ if and only if $i$ and $i+1$ are out of order in the one-line notation of $\tau$. We also set $\operatorname{des}(\tau):=|\operatorname{Des}(\tau)|$ and $\operatorname{ides}(\tau):=|\operatorname{iDes}(\tau)|$. 
In particular, $\tau(0)=0<\tau(1)$ so that 0 is never a descent or inverse descent. Any ordered subset $S=\left\{s_{1} \prec s_{2} \prec \cdots \prec s_{k}\right\} \subseteq \check{P}$ determines a subword of $\tau$

$$
\left.\tau\right|_{S}:=\tau\left(s_{1}\right) \tau\left(s_{2}\right) \ldots \tau\left(s_{k}\right)
$$

and we define $\operatorname{des}\left(\left.\tau\right|_{S}\right)$ as the number descents of the word $\left.\tau\right|_{S}$.

We call a permutation $\tau \in \mathfrak{S}_{n}$ descent-compatible with $P$ if for all $a \succ \widehat{0}$ and any saturated chain $C=\left\{\widehat{0}=c_{1} \prec \cdot c_{2} \prec \cdot \cdots \prec \cdot c_{k}=a\right\} \subseteq \breve{P}$, the number of descents $\operatorname{des}\left(\left.\tau\right|_{C}\right)$ is independent of $C$. Of course, it suffices to require this for all $a \in \max (P)$. We denote by $\operatorname{DC}(P) \subseteq \mathfrak{S}_{n}$ the descent-compatible permutations of $P$. For $\tau \in \operatorname{DC}(P)$ and $a \in P$, we write $\operatorname{des}_{P, \tau}(a)$ for the number of descents of $\operatorname{des}\left(\left.\tau\right|_{C}\right)$ for any maximal chain $C$ ending in $a$.

Theorem 3.1. Let $(P, \preceq)$ be a naturally labeled poset. Then $\mathbf{q}+\Delta_{\tau} \subseteq \mathscr{L}(P)$ for $\tau \in \mathfrak{S}_{n}$ and $\mathbf{q} \in \mathbb{Z}^{n}$ if and only if $\tau$ is descent-compatible with $P$ and $q_{a}=\operatorname{des}_{P, \tau}(a)$ for all $a \in P$.

Proof. Observe that $\mathbf{q}+\Delta_{\tau}$ is part of the alcoved triangulation of an alcoved polytope $\mathcal{P}$ if and only if $\mathbf{q}+\mathbf{c} \in \mathcal{P}$, where $\mathbf{c}$ is any point in the relative interior of $\Delta_{\tau}$. A canonical choice is the barycenter $\mathbf{c}^{\tau}$ of $\Delta_{\tau}$ given by $c_{i}^{\tau}:=\frac{\tau(i)}{n+1}$ for $i=1, \ldots, n$. Hence, we need to determine when $\mathbf{q}+\mathbf{c}^{\tau}$ satisfies the inequalities given in (1.1). Now, if $a \in P$ is a minimum, then

$$
0 \leq\left(\mathbf{q}+\mathbf{c}^{\tau}\right)_{a}=q_{a}+\frac{\tau(a)}{n+1} \leq 1
$$

and hence $q_{a}=0$. For a cover relation $a \prec \bullet b$, we calculate

$$
0 \leq q_{b}-q_{a}+\frac{\tau(b)-\tau(a)}{n+1} \leq 1 .
$$

If $\tau(b)>\tau(a)$, then this holds if $q_{b}=q_{a}$. If $\tau(b)<\tau(a)$, then $q_{b}=q_{a}+1$. Thus, $q_{b}$ is the number of descents of $\operatorname{des}\left(\left.\tau\right|_{C}\right)$ where $C=\left\{\widehat{0}=c_{1} \prec \cdot c_{2} \prec \cdot \cdots \prec \cdot c_{k}=b\right\}$ is any saturated chain.

Note that if $P=\{1, \ldots, n\}$ is totally ordered, then $\operatorname{DC}(P)=\mathfrak{S}_{n}$ and $\operatorname{des}_{P, \tau}(i)$ is the number of descents in the word $\tau(1) \cdots \tau(i)$.

Proposition 3.2. Let $P$ be a connected poset on $n$ elements. Then $\mathrm{DC}(P)=\mathfrak{S}_{n}$ if and only if $P$ is a rooted tree.

Proof. If $P$ is a rooted tree, then there is a unique saturated chain from $\widehat{0}$ to any given $b \in P$ and hence any $\tau \in \mathfrak{S}_{n}$ is trivially descent-compatible. Conversely, if there are two distinct maximal chains $C_{1}, C_{2}$ ending in $b \in P$, then it is easy to find a permutation $\tau \in \mathfrak{S}_{n}$ with $\operatorname{des}\left(\left.\tau\right|_{C_{1}}\right) \neq \operatorname{des}\left(\left.\tau\right|_{C_{2}}\right)$.

Example 3.3. We have already seen in the previous proposition that all $n$ ! permutations are descent compatible for the short rooted tree from Example 2.6. On the other hand, the descent compatible permutations for the short hanging tree are those $2(n-1)$ ! permutations of $[n]$ for which $\tau(n) \in\{1, n\}$.

If $\mathcal{P} \subset \mathbb{R}^{n}$ is a full-dimensional lattice polytope, the Ehrhart function $E(\mathcal{P}, k):=\left|k \mathcal{P} \cap \mathbb{Z}^{n}\right|$ agrees with a polynomial in $k$ of degree $n=\operatorname{dim} \mathcal{P}$ and the $\boldsymbol{h}^{*}$-polynomial of $\mathcal{P}$ is defined by

$$
h^{*}(\mathcal{P}, z)=h_{0}^{*}+h_{1}^{*} z+\cdots+h_{n}^{*} z^{d}:=(1-z)^{n+1} \sum_{k \geq 0} E(\mathcal{P}, k) z^{k} .
$$

See, for example, [1] for details. If $\mathcal{P}$ has a unimodular triangulation, then the $h^{*}$-polynomial can be computed very elegantly by means of half-open decompositions. Let $\mathcal{P}=\mathcal{P}_{1} \cup \cdots \cup \mathcal{P}_{r}$ be a dissection of $\mathcal{P}$ into (lattice) polytopes, i.e., every $\mathcal{P}_{i}$ is a full-dimensional (lattice) polytope that does not meet the interior of $\mathcal{P}_{j}$ for $j \neq i$. A point $\mathbf{w} \in \operatorname{int}(\mathcal{P})$ is in general position with respect to the dissection if $\mathbf{w}$ is not contained in the arrangement of facet-defining hyperplanes of $\mathcal{P}_{i}$ for all $i$. The point $\mathbf{w}$ is beyond a facet $F \subset \mathcal{P}_{i}$ if the facet-defining hyperplane aff $(F)$ separates $\mathbf{w}$ from the interior of $\mathcal{P}_{i}$. The half-open polytope associated to $\mathcal{P}_{i}$ and $\mathbf{w}$ is

$$
\mathbb{H}_{\mathbf{w}} \mathcal{P}_{i}:=\mathcal{P}_{i} \backslash \bigcup_{F} F
$$


where the union is over all facets $F \subset \mathcal{P}_{i}$ for which $\mathbf{w}$ is beyond.

Lemma 3.4 ([8, Thm. 3]). Let $\mathcal{P}=\mathcal{P}_{1} \cup \cdots \cup \mathcal{P}_{m}$ be a dissection and $\mathbf{w} \in \operatorname{int}(\mathcal{P})$ in general position with respect to the dissection. Then

$$
\mathcal{P}=\mathbb{H}_{\mathbf{w}} \mathcal{P}_{1} \uplus \mathbb{H}_{\mathbf{w}} \mathcal{P}_{2} \uplus \cdots \uplus \mathbb{H}_{\mathbf{w}} \mathcal{P}_{m} .
$$

In particular, if all polytopes $\mathcal{P}_{i}$ are lattice polytopes, then

$$
h^{*}(\mathcal{P}, z)=h^{*}\left(\mathbb{H}_{\mathbf{w}} \mathcal{P}_{1}, z\right)+h^{*}\left(\mathbb{H}_{\mathbf{w}} \mathcal{P}_{2}, z\right)+\cdots+h^{*}\left(\mathbb{H}_{\mathbf{w}} \mathcal{P}_{m}, z\right) .
$$

Since alcoved polytopes are invariant under lattice translations and coordinate permutations, we choose a suitable embedding before computing the $h^{*}$-vector.

Proposition 3.5. Let $\mathcal{P} \subset \mathbb{R}^{n}$ be a full-dimensional alcoved polytope. Then there is a lattice translation and a relabeling of coordinates such that $\Delta_{\mathrm{id}} \subseteq \mathcal{P} \subset \mathbb{R}_{\geq 0}^{n}$.

Proof. Let $\ell(\mathbf{x})$ be any linear function such that $\ell(\mathbf{p})>0$ for all $\mathbf{p} \in \mathbb{R}_{\geq 0}^{n}$ with $\mathbf{p} \neq 0$. Then $\mathbf{q}$ is the unique minimizer of $\ell(\mathbf{x})$ over $\mathbf{q}+\Delta_{\tau}$ for all $\mathbf{q}$ and $\tau$. Since $\mathcal{P}$ can be dissected into finitely many alcoves, this shows that there is a unique $\mathbf{q} \in \mathcal{P}$ that minimizes $\ell(\mathbf{x})$ over $\mathcal{P}$. Since this holds for all such linear functions, this shows that there is a point $\mathbf{q}_{0} \in \mathcal{P}$ that minimizes all $\ell(\mathbf{x})$ and hence $\mathcal{P} \subseteq \mathbf{q}+\mathbb{R}_{\geq 0}^{n}$. Thus, $\Delta_{\tau} \subseteq \mathcal{P}-\mathbf{q} \subset \mathbb{R}_{\geq 0}^{n}$ for some $\tau$ and relabeling the coordinates finishes the proof.

Making use of the previous result, we may compute the half-open decomposition with respect to the point $\mathbf{w}=\frac{1}{n+1}(1,2, \ldots, n)$. With these conventions, we are now ready to compute the $h^{*}$-polynomial of Lipschitz polytopes. For a naturally labeled poset $P$ and a descent-compatible permutation $\tau$, let us define $\operatorname{Ldes}_{P}(\tau)$ as the number of $i \in\{0, \ldots, n-1\}$ such that

$$
\operatorname{des}_{P, \tau}(\hat{\tau}(i))<\operatorname{des}_{P, \tau}(\hat{\tau}(i+1)) \quad \text { or } \quad\left(\operatorname{des}_{P, \tau}(\hat{\tau}(i))=\operatorname{des}_{P, \tau}(\hat{\tau}(i+1)) \text { and } i \in \operatorname{iDes}(\tau)\right)
$$

or, equivalently, as the number of pairs $(a, b) \in \check{P} \times \check{P}$ with $\tau(a)=\tau(b)-1$ and

$$
\operatorname{des}_{P, \tau}(a)<\operatorname{des}_{P, \tau}(b) \quad \text { or } \quad\left(\operatorname{des}_{P, \tau}(a)=\operatorname{des}_{P, \tau}(b) \text { and } a>b\right) .
$$

The equivalence of the two descriptions is easily seen by considering $i=\tau(a)$ and $i+1=\tau(b)$.

As $\operatorname{des}_{P, \tau}$ is by definition weakly increasing along chains in $P$, the subsets of $P$ for which $\operatorname{des}_{P, \tau}$ is constant partitions $P$ into layers. The description of $\operatorname{Ldes}_{P}(\tau)$ can then be easily read off the poset labeled by $\tau$. The following example illustrates this.

Example 3.6. We consider the following naturally labeled poset $P$ on $\{1, \ldots, 9\}$, and the permutation $\tau=423716598 \in \mathrm{DC}(P)$. The image of $\tau$ is given in big (black) while the natural labelling is given in small (blue). Moreover, we indicated the (boundaries of the) layers of $\mathbf{q}^{\tau}=(0,1,1,1,2,1,1,2,2)$ for $q_{a}^{\tau}=\operatorname{des}_{P, \tau}(a)$ in red.

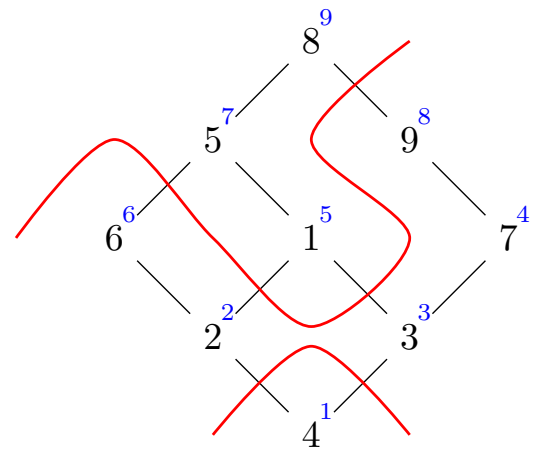

Finally,

$$
\begin{aligned}
& \left|\left\{i: \operatorname{des}_{P, \tau}(\hat{\tau}(i))<\operatorname{des}_{P, \tau}(\hat{\tau}(i+1))\right\}\right|=|\{4,7\}|=2, \\
& \left|\left\{i: \operatorname{des}_{P, \tau}(\hat{\tau}(i))=\operatorname{des}_{P, \tau}(\hat{\tau}(i+1)), i \in \operatorname{iDes}(\tau)\right\}\right|=|\{6\}|=1,
\end{aligned}
$$


or, equivalently,

$$
\begin{array}{ll}
\mid\left\{(a, b): \operatorname{des}_{P, \tau}(a)<\operatorname{des}_{P, \tau}(b),\right. & \tau(a)=\tau(b)-1\}|=|\{(1,7),(4,9)\} \mid=2, \\
\mid\left\{(a, b): \operatorname{des}_{P, \tau}(a)=\operatorname{des}_{P, \tau}(b),\right. & \tau(a)=\tau(b)-1, \quad a>b\}|=|\{(6,4)\} \mid=1 .
\end{array}
$$

We thus obtain $\operatorname{Ldes}_{P}(\tau)=3$.

Theorem 3.7. Let $(P, \preceq)$ be a naturally labeled poset. Then

$$
h^{*}(\mathscr{L}(P), z)=\mathcal{G}_{\text {Ldes }}(P, z):=\sum_{\tau \in \operatorname{DC}(P)} z^{\operatorname{Ldes}_{P}(\tau)} .
$$

In order to prove Theorem 3.7, we extract the main technical tool into the following lemma.

Lemma 3.8. Let $\tau \in \mathfrak{S}_{n}$ and $\mathbf{q}=\left(q_{1}, \ldots, q_{n}\right) \in \mathbb{Z}_{\geq 0}^{n}$. Then

$$
h^{*}\left(\mathbb{H}_{\mathbf{w}}\left(\mathbf{q}+\Delta_{\tau}\right), z\right)=z^{|A(\tau, \mathbf{q})|+|D(\tau, \mathbf{q})|},
$$

where

$$
\begin{aligned}
A(\tau, \mathbf{q}):=\{0 \leq i<n: \hat{\tau}(i)<\hat{\tau}(i+1) \quad \text { and } & \left.q_{\hat{\tau}(i)}<q_{\hat{\tau}(i+1)}\right\} \quad \text { and } \\
D(\tau, \mathbf{q}):=\{0 \leq i<n: \hat{\tau}(i)>\hat{\tau}(i+1) & \text { and } \left.\quad q_{\hat{\tau}(i)} \leq q_{\hat{\tau}(i+1)}\right\}
\end{aligned}
$$

Proof. We determine the linear inequalities of $\mathbf{q}+\Delta_{\tau}$ that are violated for $\mathbf{w}$. First observe that

$$
(\mathbf{w}-\mathbf{q})_{\hat{\tau}(n)}=\frac{\hat{\tau}(n)}{n+1}-q_{\hat{\tau}(n)} \leq 1
$$

since $q_{j} \geq 0$ for all $j$. Hence, the last inequality of $\Delta_{\tau}$ is always satisfied for $\mathbf{w}-\mathbf{q}$. For $0 \leq i<n$, we have

$$
(\mathbf{w}-\mathbf{q})_{\hat{\tau}(i)}>(\mathbf{w}-\mathbf{q})_{\hat{\tau}(i+1)} \Longleftrightarrow q_{\hat{\tau}(i+1)}+\underbrace{\frac{\hat{\tau}(i)-\hat{\tau}(i+1)}{n+1}}_{=: \delta}>q_{\hat{\tau}(i)} .
$$

If $i$ is a descent of $\hat{\tau}$, then $1>\delta>0$ and the inequality holds if and only if $q_{\hat{\tau}(i+1)} \geq q_{\hat{\tau}(i)}$. Otherwise, $i$ is an ascent of $\hat{\tau}$ and $-1<\delta<0$ and the inequality holds if and only if $q_{\hat{\tau}(i+1)}>$ $q_{\hat{\tau}(i)}$.

Proof of Theorem 3.7. By Theorem 3.1, the simplices of the alcove triangulation of $\mathscr{L}(P)$ are given by $\mathbf{q}^{\tau}+\Delta_{\tau}$ for $\tau \in \mathrm{DC}(P)$ and $q_{a}^{\tau}=\operatorname{des}_{P, \tau}(a)$ for all $a \in P$. In particular, if $P$ is naturally labeled, then $\Delta_{\text {id }} \subseteq \mathscr{L}(P) \subseteq \mathbb{R}_{\geq 0}^{n}$ and we can apply Lemma 3.8 to $\mathbb{H}_{\mathbf{w}}\left(\mathbf{q}^{\tau}+\Delta_{\tau}\right)$ and it is easy to see that $\operatorname{Ldes}_{P}(\tau)=\left|A\left(\tau, \mathbf{q}^{\tau}\right)\right|+\left|D\left(\tau, \mathbf{q}^{\tau}\right)\right|$. Lemma 3.4 then completes the proof.

If $P$ is a chain on $n$ elements, then $\operatorname{DC}(P)=\mathfrak{S}_{n}$ and the statistic given in Theorem 3.7 reduces to a known permutation statistic. For a permutation $\tau \in \mathfrak{S}_{n}$, a big ascent (or 2-ascent) is an index $0 \leq i<n$ such that $\tau(i+1)-\tau(i) \geq 2$. In particular, $i=0$ is a big ascent if and only if $\tau(1)>1$. We record the number of big ascents by $\operatorname{asc}^{(2)}(\tau)$, and $\operatorname{set} \operatorname{iasc}^{(2)}(\tau):=\operatorname{asc}^{(2)}(\hat{\tau})$ and

$$
A_{n}^{(2)}(z)=\sum_{i=0}^{n} A^{(2)}(n, i) z^{i}:=\sum_{\tau \in \mathfrak{S}_{n}} z^{\operatorname{iasc}^{(2)}(\tau)}=\sum_{\tau \in \mathfrak{S}_{n}} z^{\operatorname{asc}^{(2)}(\tau)} .
$$

Big ascents and big descents appeared in the literature before, we refer to [11] for several identities involving big descents, compare also [10, Lem. 6.5] for later reference.

Theorem 3.9. Let $P$ be a rooted tree on $n$ elements. Then

$$
\mathcal{G}_{\text {Ldes }}(P, z)=A_{n}^{(2)}(z) .
$$

Proof. We prove the result only for the case that $P$ is the chain on $n$ elements. By Proposition 2.2, $\mathscr{L}(P)$ and $\mathscr{L}\left(P^{\prime}\right)$ are lattice-equivalent whenever $P$ and $P^{\prime}$ are rooted trees on the same number of elements and hence $\mathcal{G}_{\text {Ldes }}(P, z)=\mathcal{G}_{\text {Ldes }}\left(P^{\prime}, z\right)$. 
From Theorem 3.1 we infer that for any permutation $\tau \in \mathfrak{S}_{n}, \mathbf{q}^{\tau}+\Delta_{\tau} \subset \mathscr{L}(P)$ for $q_{a}^{\tau}=\operatorname{des}_{P, \tau}(a)$. We use Lemma 3.8 for the computation. Since $P=\{1, \ldots, n\}$ is a chain, we observe that that $q_{i}^{\tau} \leq q_{j}^{\tau}$ for $i<j$ and $q_{i}=q_{j}$ if and only if there is no descent in $\tau(i) \tau(i+1) \ldots \tau(j-1)$.

Now, if $1 \leq i<n$ so that $\hat{\tau}(i+1)<\hat{\tau}(i)$, then $q_{\hat{\tau}(i+1)}^{\tau}<q_{\hat{\tau}(i)}^{\tau}$. Indeed, if $\hat{\tau}(i+1)<\hat{\tau}(i)$, then $\tau(\hat{\tau}(i+1)) \tau(\hat{\tau}(i+1)+1) \cdots \tau(\hat{\tau}(i))$ inevitably contains a descent. This implies that $D\left(\tau, \mathbf{q}^{\tau}\right)=\varnothing$.

Otherwise, $\hat{\tau}(i+1)>\hat{\tau}(i)$ and hence $q_{\hat{\tau}(i)}^{\tau} \leq q_{\hat{\tau}(i+1)}^{\tau}$ with equality if and only if we have $\operatorname{Des}(\tau) \cap$ $\{\hat{\tau}(i), \ldots, \hat{\tau}(i+1)-1\}=\varnothing$. But this is the case if and only if $\hat{\tau}(i+1)=\hat{\tau}(i)+1$. Therefore, $q_{\hat{\tau}(i)}^{\tau}<q_{\hat{\tau}(i+1)}^{\tau}$ if and only if $\hat{\tau}(i+1) \geq \hat{\tau}(i)+2$. This gives $\operatorname{Ldes}_{P}(\tau)=\operatorname{iasc}^{(2)}(\tau)$ in the case of the $n$-chain, which yields the statement.

In light of Proposition 2.2 and the fact that the coefficients of the $h^{*}$-polynomial of the cube are given by Eulerian numbers, Theorem 3.9 implies the following corollary.

Corollary 3.10. Let $P$ be a rooted tree on $n$ elements. Then

$$
\mathcal{G}_{\text {Ldes }}(P, z)=A_{n}^{(2)}(z)=\sum_{\tau \in \mathfrak{S}_{n}} z^{\operatorname{ides}(\tau)} .
$$

We illustrate Theorem 3.7 with the computation of the $h^{*}$-polynomial for the Lipschitz polytope of short rooted and hanging trees.

Example 3.11. Using the description of $\operatorname{Ldes}_{P}$ given in (3.1) in the case of the short rooted and hanging trees from Example 2.6, the following yields

$$
\operatorname{Ldes}_{P}(\tau)=\operatorname{ides}(\tau) \text { for } \tau \in \operatorname{DC}(P)
$$

in these two cases. For the short rooted tree we have

$$
\begin{aligned}
& \left\{i: \operatorname{des}_{P, \tau}(\hat{\tau}(i))<\operatorname{des}_{P, \tau}(\hat{\tau}(i+1))\right\}=\left\{\begin{array}{l}
\varnothing \\
\{0\}
\end{array}\right. \\
& \left\{i: \operatorname{des}_{P, \tau}(a)=\operatorname{des}_{P, \tau}(b) \quad i \in \operatorname{iDes}(\tau)\right\}=\left\{\begin{array}{ll}
\operatorname{iDes}(\tau) & \text { if } \tau(1)=1 \\
\operatorname{iDes}(\tau) \backslash\{\tau(1)-1\} & \text { if } \tau(1)>1
\end{array} .\right.
\end{aligned}
$$

On the other hand, for the short hanging tree, we have $\tau(n) \in\{1, n\}$ and two analogous considerations for these two possibilities.

Even though one easily finds examples of posets $P$ and permutations $\tau \in \operatorname{DC}(P)$ for which $\operatorname{Ldes}_{P}(\tau) \neq \operatorname{ides}(\tau)$, the generating function search functionality of the Combinatorial Statistic Finder www. FindStat .org [12] suggests the following conjectural generalization of Corollary 3.10 to all posets, in agreement with Example 3.11.

Conjecture 3.12. Let $P$ be a poset. Then

$$
\mathcal{G}_{\text {Ldes }}(P, z)=\sum_{\tau \in \operatorname{DC}(P)} z^{\operatorname{ides}(\tau)} .
$$

\section{RANKED POSETS}

A poset $P$ is ranked, if for all $a, b \in P$, every maximal chain in $[a, b]_{P}=\{c \in P: a \preceq c \preceq b\}$ has the same length. If $P$ has a minimal element, then this is equivalent to the existence of a rank function $\rho: P \rightarrow \mathbb{Z}_{\geq 0}$ such that $\rho(\widehat{0})=0$ and $\rho(b)=\rho(a)+1$ for $a \prec \cdot b$.

Proposition 4.1. If $P$ is a poset such that $\breve{P}$ is ranked, then $\mathscr{L}(P)$ is centrally-symmetric with respect to $\rho$, i.e., $\rho-\mathscr{L}(P)=\mathscr{L}(P)$.

Proof. Simply note that $\rho(b)-\rho(a)-(f(b)-f(a))=1-(f(b)-f(a))$ for all $\widehat{0} \preceq a \prec \cdot b$. 
A lattice polytope $\mathcal{P} \subset \mathbb{R}^{n}$ is reflexive if $\mathbf{0}$ is the only lattice point in the interior and the polar polytope is again a lattice polytope. A lattice polytope $\mathcal{P}$ is $\boldsymbol{r}$-Gorenstein for some $r \in \mathbb{Z}_{>0}$ if $r \mathcal{P}$ contains a unique lattice point $\mathbf{q}$ and $r \mathcal{P}-\mathbf{q}$ is reflexive.

Proposition 4.2. If $P$ is a poset such that $\breve{P}$ is ranked, then $\mathscr{L}(P)$ is 2-Gorenstein.

Proof. From (1.1), it is clear that $f \in \mathbb{Z}^{P}$ is in the interior of $2 \mathscr{L}(P)$ if $f(a)=1$ for all $a \in \min (P)$ and $f(b)=f(a)+1$ for all $a \prec \cdot b$. Hence, $f$ is a rank function, which is unique whenever $\breve{P}$ is ranked. The polytope $2 \mathscr{L}(P)-\rho$ is given by $-1 \leq f(b)-f(a) \leq 1$ for all $\widehat{0} \preceq a \prec \cdot b$, which implies that the polar polytope is a lattice polytope with vertices $\pm \mathbf{1}_{a}$ and $\pm\left(\mathbf{1}_{b}-\mathbf{1}_{a}\right)$.

Stanley [18] noted that, in the case that an $n$-dimensional polytope $\mathcal{P}$ is $r$-Gorenstein, one has $h_{i}^{*}(\mathcal{P})=h_{n+1-r-i}^{*}(\mathcal{P})$ for all $r$. Bruns-Römer [2] showed that if an $r$-Gorenstein polytope has a regular and unimodular triangulation, then the $h^{*}$-vector is unimodal. For Lipschitz polytopes, such a triangulation is vouched for by Theorem 3.1 and with Proposition 4.2, we get the following.

Theorem 4.3. Let $P$ be a poset on $n$ elements such that $\breve{P}$ is ranked and let

$$
\mathcal{G}_{\text {Ldes }}(P, z)=g_{0}(P)+g_{1}(P) z+\cdots+g_{n-1}(P) z^{n-1}=\sum_{\tau \in \operatorname{DC}(P)} z^{\operatorname{Ldes}_{P}(\tau)} .
$$

Then

$$
g_{i}(P)=g_{n-1-i}(P) \quad \text { and } \quad g_{i}(P) \leq g_{i+1}(P) \text { for } i \leq\left\lfloor\frac{n-1}{2}\right\rfloor .
$$

It should be noted that if $\breve{P}$ is ranked, then $\mathrm{DC}(P)$ is invariant under reversals, i.e., if $\tau \in \mathrm{DC}(P)$ then $\bar{\tau} \in \mathrm{DC}(P)$ where $\bar{\tau}(i):=n+1-\tau(i)$. However, this symmetry does not induce the symmetry of the statistic.

Open problem 4.4. Give a combinatorial proof of Theorem 4.3.

For posets such that $\check{P}$ is ranked, the Lipschitz polytope $\mathscr{L}(P)$ can also be constructed in a different way, that explains the central-symmetry as well as the Gorenstein property. If $\breve{P}$ is ranked, then the order cone $\mathcal{K}(P)$ is a Gorenstein cone, that is, there is a unique point $\mathbf{q} \in \mathcal{K}(P)$ such that $\operatorname{int}(\mathcal{K}(P)) \cap \mathbb{Z}^{P}=(\mathbf{q}+\mathcal{K}(P)) \cap \mathbb{Z}^{P}$. Hibi [6] showed that $\mathcal{K}(P)$ is Gorenstein if and only if $\check{P}$ is ranked and hence $\mathbf{q}=\rho$. We thus get the following description of $\mathscr{L}(P)$.

Corollary 4.5. Let $P$ be a poset such that $\breve{P}$ is ranked with rank function $\rho$. Then

$$
\mathscr{L}(P)=\mathcal{K}(P) \cap(-\mathcal{K}(P)+\rho) .
$$

In this case $\mathscr{L}(P)$ is a spindle in the sense of Santos [13].

\section{P-Hypersimplices}

Let $(P, \preceq)$ be a poset with a unique maximal element $\widehat{1}$. Let ht $(P)$ be the number of elements in a maximal chain in $P$, the height of $P$. For $1 \leq k \leq h t(P)$, we define the $(\boldsymbol{P}, \boldsymbol{k})$-hypersimplex as

$$
\Delta(P, k):=\{f \in \mathscr{L}(P): k-1 \leq f(\widehat{1}) \leq k\} .
$$

The $(P, k)$-hypersimplices are again lattice polytopes and, in fact, alcoved and we can refine the description of vertices of Proposition 2.5.

Proposition 5.1. The vertices of $\Delta(P, k)$ correspond exactly to neighbor-closed chains of nonempty filters in $P$ of length $k-1$ or $k$.

In particular, this gives a nice interpretation of the first $(P, k)$-hypersimplex. 
Corollary 5.2. For every poset $P$ with $\widehat{1}, \Delta(P, 1)$ is the order polytope $\mathcal{O}(P)$. Moreover, if $\check{P}$ is ranked, then

$$
\Delta(P, k) \cong \Delta(P, \operatorname{ht}(P)+1-k),
$$

for $1 \leq k \leq \mathrm{ht}(P)$.

In the case that $P$ is the $n$-chain, $\Delta(P, k)$ recovers, after the transformation used already in Example 2.1, the well-known $(\boldsymbol{n}, \boldsymbol{k})$-hypersimplex, introduced in [4],

$$
\begin{aligned}
\Delta(n, k) & :=\operatorname{conv}\left\{\mathbf{y} \in\{0,1\}^{n+1}: y_{1}+\cdots+y_{n+1}=k\right\} \\
& \cong \quad\left\{\mathbf{y} \in[0,1]^{n}: k-1 \leq y_{1}+\cdots+y_{n} \leq k\right\} .
\end{aligned}
$$

We can define the notion of $\boldsymbol{P}$-descents on $\operatorname{DC}(P)$ by $\operatorname{des}_{P}(\tau):=\operatorname{des}_{P, \tau}(\widehat{1})$ for $\tau \in \operatorname{DC}(P)$.

Corollary 5.3. Let $P$ be a poset on $n$ elements with $\widehat{1}$. Then, for $1 \leq k \leq \operatorname{ht}(P)$,

$$
n ! \cdot \operatorname{vol} \Delta(P, k)=\left|\left\{\tau \in \operatorname{DC}(P): \operatorname{des}_{P}(\tau)=k-1\right\}\right| .
$$

Corollary 5.3 is a is a direct consequence of Theorem 3.1 and generalizes of the well-known result for $P$ being an $n$-chain: The volume of $\Delta(n+1, k+1)$ normalized by $n$ ! is the Eulerian number $A(n, k)$. This result is attributed to Laplace and was proved by geometric means in [16]; see also $[9,10]$.

The central-symmetry of $\mathscr{L}(P)$ in the case that $\breve{P}$ is ranked (Proposition 4.1), yields the following symmetry of $P$-descents, generalizing the classical symmetry of the descent statistic.

Corollary 5.4. Let $P$ be a poset with $\widehat{1}$ such that $\check{P}$ is ranked. Then for $1 \leq k \leq \operatorname{ht}(P)$

$$
\left|\left\{\tau \in \mathrm{DC}(P): \operatorname{des}_{P}(\tau)=k-1\right\}\right|=\left|\left\{\tau \in \mathrm{DC}(P): \operatorname{des}_{P}(\tau)=\operatorname{ht}(P)-k\right\}\right| .
$$

The short hanging trees of Example 2.6 are exactly the connected posets with $\widehat{1}$ of height 2 . Corollary 5.2 yields that in this case $\mathscr{L}(P)$ can be decomposed into two congruent copies of $\mathcal{O}(P)$. The following combinatorial implication can be seen also directly from Example 3.11.

Corollary 5.5. Let $(P, \preceq)$ be a short hanging tree on $n$ elements. Then

$$
h^{*}(\mathscr{L}(P), z)=(1+z) \cdot h^{*}(\mathcal{O}(P), z) .
$$

Li [10] gave an interpretation of the $h^{*}$-vector of the half-open hypersimplices

$$
\Delta^{\mathbf{\omega}}(n, k+1):=\left\{\mathbf{y} \in[0,1]^{n}: k<y_{1}+\cdots+y_{n} \leq k+1\right\},
$$

for $1 \leq k<n$. Using generating functions and shellings of the alcoved triangulation, the following was shown.

Theorem 5.6 ([10, Thm. 1.3]).

$$
h^{*}\left(\Delta^{\boldsymbol{\omega}}(n, k+1), z\right)=\sum_{\substack{\tau \in \mathfrak{S}_{n} \\ \operatorname{des}(\tau)=k}} z^{\operatorname{iasc}^{(2)}(\tau)} .
$$

Since the alcoved triangulation of $\mathscr{L}(P)$ is compatible with restriction to the half-open $(P, k)$ hypersimplices

$$
\Delta^{\mathbf{\top}}(P, k+1):=\{f \in \mathscr{L}(P): k<f(\widehat{1}) \leq k+1\},
$$

we obtain the following generalization of Theorem 5.6, which is the case that $P$ is the $n$-chain.

Corollary 5.7. Let $P$ be a finite poset and $1 \leq k \leq h(P)$. Then

$$
h^{*}\left(\Delta^{\boldsymbol{\bullet}}(P, k), z\right)=\sum_{\substack{\tau \in \operatorname{DC}(P) \\ \operatorname{des}(\tau)=k}} z^{\operatorname{Ldes}_{P}(\tau)} .
$$




\section{REFERENCES}

[1] M. Beck and S. Robins, Computing the Continuous Discretely: Integer-point Enumeration in Polyhedra, Undergraduate Texts in Mathematics, Springer, New York, 2007. 5

[2] W. Bruns and T. Römer, h-vectors of Gorenstein polytopes, J. Combin. Theory Ser. A, 114 (2007), pp. 6576. 9

[3] T. Chappell, T. Friedl, and R. Sanyal, Two double poset polytopes. Preprint, June 2016, 35 pages, arXiv:1606.04938. 4

[4] I. M. Gel'fand, R. M. Goresky, R. D. MacPherson, and V. V. Serganova, Combinatorial geometries, convex polyhedra, and Schubert cells, Adv. in Math., 63 (1987), pp. 301-316. 2, 10

[5] J. Gouveia, P. A. Parrilo, and R. R. Thomas, Theta bodies for polynomial ideals, SIAM J. Optim., 20 (2010), pp. 2097-2118. 4

[6] T. HiBI, Distributive lattices, affine semigroup rings and algebras with straightening laws, in Commutative algebra and combinatorics (Kyoto, 1985), vol. 11 of Adv. Stud. Pure Math., North-Holland, Amsterdam, 1987, pp. 93-109. 9

[7] S. Hopkins And D. Perkinson, Bigraphical arrangements, Trans. Amer. Math. Soc., 368 (2016), pp. 709725. 2

[8] M. Köppe and S. Verdoolaege, Computing parametric rational generating functions with a primal Barvinok algorithm, Electron. J. Comb in., 15 (2008). 6

[9] T. Lam And A. Postnikov, Alcoved polytopes. I, Discrete Comput. Geom., 38 (2007), pp. 453-478. 4, 10

[10] N. LI, Ehrhart $h^{*}$-vectors of hypersimplices, Discrete Comput. Geom., 48 (2012), pp. 847-878. 2, 7, 10

[11] T. Mansour, M. Shattuck, And D. G. L. Wang, Counting subwords in flattened permutations, J. Comb., 4 (2013), pp. $327-356.7$

[12] M. Rubey, C. Stump, et Al., FindStat: The Combinatorial Statistic Finder. www.FindStat.org, 2017. 8

[13] F. Santos, A counterexample to the Hirsch conjecture, Ann. of Math. (2), 176 (2012), pp. 383-412. 9

[14] R. Sanyal, A. Werner, And G. M. Ziegler, On Kalai's conjectures concerning centrally symmetric polytopes, Discrete Comput. Geom., 41 (2009), pp. 183-198. 4

[15] J. Y. SHI, The Kazhdan-Lusztig cells in certain affine Weyl groups, vol. 1179 of Lecture Notes in Mathematics, Springer-Verlag, Berlin, 1986. 2

[16] R. P. Stanley, Eulerian partitions of a unit hypercube, in Higher Combinatorics, M. Aigner, ed., D. Reidel Publishing Co., Dordrecht-Boston, Mass., 1977. 10

[17] — Two poset polytopes, Discrete Comput. Geom., 1 (1986), pp. 9-23. 1

[18] _ Combinatorics and commutative algebra, vol. 41 of Progress in Mathematics, Birkhäuser Boston, Inc., Boston, MA, second ed., 1996. 9

[19] S. Sullivant, Compressed polytopes and statistical disclosure limitation, Tohoku Math. J. (2), 58 (2006), pp. $433-445.4$

[20] The Sage Developers, SageMath, Mathematics Software System (Ver. 7.5), 2017. www. SageMath.org. 3

Institut für Mathematik, Goethe-Universität Frankfurt, Germany

E-mail address: sanyal@math.uni-frankfurt.de

Institut für Mathematik, Freie Universität Berlin, Germany

E-mail address: christian.stump@fu-berlin.de 